\title{
Introduction of laparoscopy-assisted distal gastrectomy: a tale of two cities
}

\author{
Yasuhiro Kodera
}

Published online: 9 May 2012

(c) The International Gastric Cancer Association and The Japanese Gastric Cancer Association 2012

The most frequent type of surgery for gastric cancer in Japan is distal gastrectomy, due to the predominantly distal origin of the tumors and the relatively early stages at which they are diagnosed. As it is technically less challenging than total gastrectomy, an increasing number of surgeons are currently in the process of adding laparoscopy-assisted distal gastrectomy (LADG) to their armamentarium. Not long ago, there were only a handful of surgeons that spoke on this subject at domestic surgical meetings. These surgeons attempted LADG with instruments and scopes that were not as sophisticated as the devices we have today. It is advisable, nowadays, to use the best devices and instruments available, and-even more importantly-to seek assistance and advice from an experienced laparoscopic surgeon when performing the LADG as a beginner. That brings us to the question of who taught these "first-generation" laparoscopic surgeons when they were beginners. The reality is that these highly-motivated "beginners" took the risk of creating a completely new type of surgery, assisted by less capable colleagues, using suboptimal devices, and with no one to seek for advice. There could have been casualties, due to either surgical complications or oncologically inadequate manipulation, and one can easily imagine that not all of these pioneers achieved the goal they wanted. It is important to note that those who were successful in their early attempts at LADG had all been (and still are) tremendously skillful at open surgery,

This editorial refers to the article doi:10.1007/s10120-012-0157-2.

Y. Kodera $(\square)$

Department of Gastroenterological Surgery (Surgery II),

Nagoya University Graduate School of Medicine,

65 Tsurumai-cho, Showa-ku, Nagoya, Aichi 466-8550, Japan

e-mail: ykodera@med.nagoya-u.ac.jp with supreme knowledge of anatomy and meticulous surgical technique. In addition, they probably felt comfortable when they performed laparoscopic cholecystectomy for the first time, and may have had an instinct that they could perform more complex surgical procedures using this new approach.

By the first decade of the twenty-first century, a substantial proportion of the videos featuring gastric cancer surgery at domestic surgical meetings were performed by a laparoscopic approach. Unlike the "first generation" surgeons, younger experts in this field must have had a greater chance of interacting with or learning from more experienced surgeons. Thus, aside from welcoming an established laparoscopic surgeon as a new staff member, there were other ways of introducing the new approach to a surgical team with no prior experience of LADG.

More recently, two leading cancer centers in Japan, one in Nagoya and another in Yokohama, introduced LADG through a multistep training system planned by the staff surgeons of each institution and conducted after the approval of the corresponding institutional review board. Mochizuki et al. [1] described their experience at Aichi Cancer Center, Nagoya, in a recent issue of the journal Gastric Cancer. Two staff surgeons who were board-certified by the Japan Surgical Society, who had performed at least 50 open gastrectomies with lymph node dissection, and who had experienced >30 laparoscopic cholecystectomies during their careers as general surgeons participated in this program as trainees. They had some training both in the dry laboratory and with porcine models, and visited Nagoya University to observe LADG operated by Dr. Fujiwara, one of the "first-generation" laparoscopic surgeons [2]. The initial 10 LADGs at Aichi Cancer Center were then performed with either of the two surgeons as an operator and Dr. Fujiwara as an assistant, while the other 
surgeon handled the laparoscope to share the experience. After the initial 10 cases, the two surgeons performed a further 50 cases together, with one surgeon invariably taking the role of assistant while the other was operating. The morbidity rate during the training period was $2 \%$, and there was no mortality. Three cases were converted to open surgery, one due to injury of the mesocolon and the other two due to oncological reasons. More blood loss occurred in the earlier 25 cases, but no significant differences in other parameters were observed between the earlier and later groups.

In the Yokohama district, three surgeons at three independent hospitals, including Kanagawa Cancer Center, became trainees at their institutions in another training program featured in the current issue of Gastric Cancer [3]. Each surgeon sought assistance from another "first-generation" laparoscopic surgeon, but on this occasion only for the first three cases. Prior to this challenging experience, the three surgeons had performed $>300$ open gastrectomies, $>100$ laparoscopic cholecystectomies, $>5$ laparoscopic colectomies, and $>5$ laparoscopic wedge resections of the stomach. They also had some training in both the dry and wet laboratories, but-more importantly-they took substantial time to scrutinize and learn from the videos of exemplarily conducted LADGs. This, Dr. Yoshikawa et al. considers, was a key component of the training that may have been lacking from the Nagoya experience. With that much experience, and after that much effort, the authors declared that LADG was only a few steps away from open gastrectomy. Indeed, it is important to check the video after surgery to see which part of the surgery fell short of being ideally conducted.

On the other hand, profound knowledge of the surgical procedures per se does not guarantee that LADG can be conducted safely. Surgeons must be trained to handle the instruments with sufficient accuracy and dexterity, a task that usually cannot be achieved without moving their own hands. Training sessions with a dry box, virtual simulators, and live animals are therefore mandatory. Even with costly virtual reality simulators, it remains unclear whether an improvement in hand-eye coordination, as quantitated by the device, really does translate into success in a surgery as complex as LADG [4]. However, the transfer validity of these simulators is likely to improve as a response to increasing demand in the market. This editor is rather unsatisfied that the amount of training that should be assigned to the trainees in this aspect was not clearly defined in either the Nagoya or the Yokohama experience. Perhaps the amount of training needed to acquire the necessary skills depends on the inherent ability of the trainees and therefore cannot be defined. At the same time, one must be realistic regarding the time and cost that can be expended on such training, given that the trainees in both projects were, at the same time, full-time staff surgeons of prominent hospitals. The three surgeons in Yokohama seemed to be comfortable with performing LADG after only three site visits by the experts. This editor is left with amazement-along with some skepticism-that they may have been so exceptionally talented. A great deal of time, effort, and cost would be required to train a less talented trainee; whether such a trainee could be identified quantitatively using simulators and then discouraged from pursuing a career as a surgeon are important issues for future debate.

Both Dr. Mochizuki and Dr. Yoshikawa were subsequently qualified by the Japan Society for Endoscopic Surgery as instructing surgeons in this field after scrutiny of their unedited videos through a stringent review system. Not many surgeons have had such glory and, with this tremendous outcome, the experiences at Nagoya and Yokohama can both be considered successes. Can such training programs be provided for all Japanese institutions? One advantage the colleagues at the two cancer centers had over other general surgeons was that they belonged to celebrated high-volume hospitals as staff members. Even if they selected patients strictly in terms of body mass index and other factors that may influence the short-term outcome of LADG, they did not have to wait long for the next opportunity after each fruitful experience. This advantage would apply even more prominently to institutions in Korea, where most patients with gastric cancer are treated at super-high-volume centers that conduct $>1000$ gastrectomies per a year. When the results of various on-going phase III trials are found to be positive [5, 6], it would be much easier in South Korea than in other countries to declare formally (in the guidelines, for instance) that LADG is the standard of care. In Japan, an increasing number of young surgeons are now being trained in some high-volume training centers with their own training programs $[7,8]$. Institutions that fail to employ graduates of these highly regarded programs should do their best to follow the steps of Drs. Mochizuki and Yoshikawa and attempt to convert an expert in open surgery into a capable laparoscopic surgeon.

The day will eventually come when the first gastric cancer surgery in a young surgeon's career needs to be performed by the laparoscopic approach. Is it possible that the many years we spent as residents learning to perform open surgery will be simply dismissed as unnecessary for the younger generation? It is painful to answer in the affirmative, but we must move forward. We should perhaps remember how, several decades ago, we suffered as a second assistant aiding a senior surgeon in a low anterior resection. With a retractor in each hand, we stood between the thighs of a patient for several hours to ensure that the operator and his first assistant could proceed with their 
surgery while we could see nothing and gradually became tired and frustrated. Surgical residents can now see exactly what the operator is looking at through the monitors with little need for strength and durability. What happened during surgery can easily be reproduced in a video, and scrutinizing this video would probably be more informative than reading textbooks on surgical technique with plenty of figures, photos, and written explanations. Moreover, we have allowed our medical students to perform cholecystectomy in a virtual reality simulator, and noticed that several students excel in hand-eye coordination without any prior training, possibly because they have been playing computer games throughout their childhood. It has actually been reported that prior experience with open surgery does not help when attempting to gain proficiency in laparoscopic cholecystectomy in a simulated setting [9]. While the experiences at Nagoya and Yokohama could be used to convert an established open surgeon into a laparoscopic surgeon, we should perhaps begin to construct a training program to turn a medical student with no prior experience in open surgery into a laparoscopic surgeon.

\section{References}

1. Mochizuki Y, Kodera Y, Fujiwara M, Ito Y, Misawa K, Kanemitsu Y, Ito S. Single-institute prospective trial of laparoscopy-assisted distal gastrectomy with systemic lymph node dissection for early gastric carcinoma. Gastric Cancer. 2012;15:124-30.

2. Fujiwara M, Kodera Y, Kasai Y, Kanyama Y, Hibi K, Ito K, et al. Laparoscopy-assisted distal gastrectomy with systemic lymph node dissection for early gastric carcinoma. J Am Coll Surg. 2003;196:75-81.

3. Yoshikawa T, Cho H, Rino Y, Yamamoto Y, Kimura M, Fukunaga $\mathrm{T}$, et al. A prospective feasibility study and safety study of laparoscopy-assisted distal gastrectomy for clinical stage I gastric cancer initiated by surgeons with much experience of open gastrectomy and laparoscopic surgery. Gastric Cancer (in press).

4. Iwata N, Fujiwara M, Kodera Y, Tanaka C, Ohashi N, Nakayama $\mathrm{G}$, et al. Construct validity of the LapVR virtual-reality surgical simulator. Surg Endosc. 2011;25:423-8.

5. Kim HH, Hyung WJ, Cho GS, Kim MC, Han SU, Kim W, et al. Morbidity and mortality of laparoscopic gastrectomy versus open gastrectomy for gastric cancer: an interim report: a phase III multicenter, prospective, randomized trial (KLASS Trial). Ann Surg. 2010;251:417-20.

6. Park DJ, Han SU, Hyung WJ, Kim MC, Kim W, Ryu SY, et al. Long-term outcomes after laparoscopy-assisted gastrectomy for advanced gastric cancer: a large-scale muticenter retrospective study. Surg Endosc (in press).

7. Tokunaga M, Hiki N, Fukunaga T, Miki A, Nunobe S, Ohyama S, et al. Quality control and educational value of laparoscopy-assisted gastrectomy in a high-volume center. Surg Endosc. 2009;23: 289-95.

8. Kinoshita T, Kanehira E, Matsuda M, Okazumi S, Katoh R. Effectiveness of a team participation training course for laparoscopy-assisted gastrectomy. Surg Endosc. 2010;24:561-6.

9. Brown DC, Miskovic D, Tang B, Hanna GB. Impact of established skills in open surgery on the proficiency gain process for laparoscopic surgery. Surg Endosc. 2010;24:1240-6. 